University of Nebraska - Lincoln

DigitalCommons@University of Nebraska - Lincoln

Mechanical \& Materials Engineering Faculty

Publications

Mechanical \& Materials Engineering

Department of

2020

\title{
Low Velocity Impact of Hybrid Stacked Steel Plates
}

\author{
H. Hadidi \\ University of Nebraska-Lincoln and Jazan University \\ R. Q. Feng \\ University of Nebraska-Lincoln, rfeng1@unl.edu \\ M. P. Sealy \\ University of Nebraska-Lincoln, sealy@unl.edu
}

Follow this and additional works at: https://digitalcommons.unl.edu/mechengfacpub

Part of the Mechanics of Materials Commons, Nanoscience and Nanotechnology Commons, Other Engineering Science and Materials Commons, and the Other Mechanical Engineering Commons

Hadidi, H.; Feng, R. Q.; and Sealy, M. P., "Low Velocity Impact of Hybrid Stacked Steel Plates" (2020). Mechanical \& Materials Engineering Faculty Publications. 623.

https://digitalcommons.unl.edu/mechengfacpub/623

This Article is brought to you for free and open access by the Mechanical \& Materials Engineering, Department of at DigitalCommons@University of Nebraska - Lincoln. It has been accepted for inclusion in Mechanical \& Materials Engineering Faculty Publications by an authorized administrator of DigitalCommons@University of Nebraska Lincoln. 
Published in International Journal of Impact Engineering 140 (2020) 103556; doi: 10.1016/j.ijimpeng.2020 .103556

Copyright (C 2020 Elsevier Ltd. Used by permission.

Submitted August 9, 2019; revised December 26, 2019; accepted February 27, 2020; published online February 28, 2020.

\title{
Low Velocity Impact of Hybrid Stacked Steel Plates
}

\author{
H. Hadidi, ${ }^{1,2}$ R.Q. Feng, ${ }^{1}$ and M. P. Sealy ${ }^{1}$ \\ 1. Department of Mechanical and Materials Engineering, University of Nebraska-Lincoln, Lincoln, \\ Nebraska, USA \\ 2. Department of Mechanical Engineering, Jazan University, Gizan, Jazan, Saudi Arabia \\ Corresponding author - M. P. Sealy, email sealy@unl.edu
}

\begin{abstract}
Designing and manufacturing high-strength, low-weight parts with enhanced impact resistance is highly sought after in the transportation industry. The most common methods to improve strengthto-weight ratios of impact targets is a composite material composition or a favorable geometric design. An alternative method to improve impact performance is functionally gradient mechanical properties in a single material by hybrid additive stacking. In this study, low-velocity impact tests were conducted on hybrid stacked 1070 steel plates where individual layers were subjected to shot peening (SP) to functionally grade mechanical properties. Hybrid additive stacking refers to secondarily processing preferential layers within a stacked build volume by cold working to achieve favorable compressive residual stresses and localized work hardening. Incorporating SP on preferential layer intervals during stacking is a radically different approach to increase the strength-to-weight ratio and impact performance of metals. Cold working individual layers by peening achieves functionally gradient mechanical properties in a single material without the need for multimaterial composite manufacturing. The objective of this work was to investigate the impact strength and energy absorption from stacking shot-peened and non-shot-peened layers to form a hybrid target. Identifying favorable stacking sequences provides insight on how to design a hybrid structure that incorporates a mechanical surface treatment (e.g., shot peening) to outperform conventional and composite targets. Results showed energy absorption improved by incorporating stacked shot-peened layers and was dependent on the sequence. The improvement in impact performance was attributed to the shot-peening induced compressive residual stresses and increased friction incorporated within predefined layers.
\end{abstract}

Keywords: impact, peening, hybrid manufacturing, design 


\section{Introduction}

Designing and manufacturing components with the ability to absorb energy while also providing high strength and low weight is a current challenge. The transportation industry is seeking advanced manufacturing and materials technologies that reduce the total lifecycle energy cost of fabricated metal products while providing impact resistance. Transportation components are routinely exposed to impact events from severe weather. The primary goal for transportation components is to design and manufacture lightweight structures that better minimize impact damage while protecting users and occupants. It is important that damage below a predefined threshold does not shorten the part or product's lifecycle.

The most effective method to reduce energy consumption is to decrease weight. One approach to decreasing weight while improving impact performance is the use of metalpolymer composites [1]. However, manufacturing challenges associated with injection molding along with substandard mechanical properties limits current use. Another approach for lightweighting is sandwiched honeycomb structures [2]. Structural performance and integrated honeycomb assemblies remain a manufacturing challenge [3]. Further, the 2.5-dimensional aspects of honeycomb structures introduces design limitations that hinders performance. Additive manufacturing (i.e., 3D printing) circumvents most design and manufacturing challenges. Tools such as topology optimization coupled with additive manufacturing have proven effective for light weighting. However, mechanical properties and subsequent impact performance are print "recipe" dependent. An alternative approach to improve impact performance in the transportation industry that avoids challenges from integrating composite materials, honeycomb structures, and print recipe dependence is a hybrid manufacturing technique that combines the concept of layer-based manufacturing with secondary mechanical surface treatments [4].

Producing parts with the desired impact properties can be achieved using the concept of an alternating layer sequence of different mechanical properties, which results in a functionally gradient material (FGM) [5-10]. These FGMs have the capability to perform better under impact and ballistic environments because of their enhanced mechanical properties (e.g., elasticity, toughness, fracture strength, and energy absorption) throughout the part's volume. A functionally graded target can absorb more energy before damage and failure occurs [11]. The challenge becomes merging the objectives of reducing energy consumption with improving impact performance using next generation design and manufacturing techniques.

The innovation in this work is a novel hybrid additive stacking method to fabricate high strength-to-weight ratio impact materials that improve fuel efficiency in the transportation industry. This hybrid approach couples layer-based manufacturing with surface treatment technology to produce favorable 3D mechanical properties throughout the entire build volume. Imagine being able to manufacture parts with the exact mechanical properties desired, layer by layer, without needing a secondary material or complex internal geometry.

One promising surface treatment is shot peening (SP). SP plastically deforms metals with a stochastically dispersed stream of high-velocity beads that improve strength and 
hardness. Peening has proven effective at changing surface integrity in components to enhance fatigue life and corrosion resistance [12-14]. However, the influence of shot peening on impact resistance and energy absorption has not been fully explored because of limited penetration depth as an external surface treatment. The beneficial effects of shot peening typically extend only a few millimeters below the surface. With hybrid additive stacking, shot peening can be performed on preferential layer intervals to manipulate the global impact response of a target. Application of peening throughout the build process permits tailoring the interior material characteristics of the structure to increase performance without the need for postprocessing. Better impact protection is feasible by varying the target material's elasticity, toughness, fracture strain, and energy absorption and has been demonstrated in polymers $[15,16]$.

The objective of this work was to investigate the influence of stacking sequence of SP and non-SP layers on a hybrid target's impact strength and energy absorption. The primary hypothesis of the proposed work is that parts manufactured by hybrid stacking have increased energy absorption and decreased peak force compared to a nonhybrid target. The measure of success will be a quantifiable change in the peak force and restitution. This investigation will provide insight on how to design a hybrid manufacturing process that incorporates a mechanical surface treatment and identifies a stacking sequence that performs better when subjected to low-velocity impacts. This work examines drop-weight impact tests on stacked shot-peened 1070 steel Almen strip targets. The impact behavior of this particular material with different stacking sequences were then analyzed from an energy perspective by means of two metrics: impact acceleration and restitution. The dropweight impact tests were conducted choosing one level of impact velocity and impactor mass.

\section{Experimental procedure}

An experimental investigation on nonuniform, low-velocity impact of stacked 1070 steel Almen strip plates (Fig. 1) was conducted to determine the influence of integrating shotpeened layers within the stacking sequence on energy absorption. It was hypothesized that the compressive residual stresses and work hardening provided by the shot-peened layers would increase the energy absorption of a hybrid-stacked target. Almen strips are standardized plates used to quantify the intensity of shot peening.

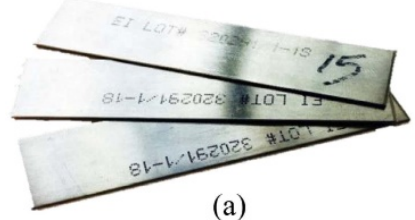

(a)

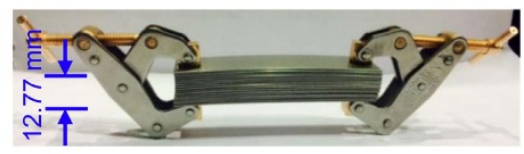

(b)

Figure 1. 1070 steel Almen strips: (a) individual strips and (b) stacked and clamped strips with intermittent peened layers. 
It is important to mention that stacked Almen strips containing shot-peened layers were not subjected to thermal cancellation from the addition of subsequent layers, as would be the case in additive manufacturing. That is, none of the residual stresses or work hardening imparted by shot peening was relaxed from heat generated by adding subsequent layers on a previously shot-peened layer. Thermal cancellation of favorable mechanical properties using this approach in additive manufacturing is expected to influence the energy absorption capability [17].

\subsection{Shot peening}

Shot peening (SP) is a localized cold-working surface treatment used mainly to improve the fatigue life of metallic components. SP impinges a target with stochastically dispersed, high-velocity steel or ceramic beads. The impact pressure is sufficient to cause plastic deformation that results in compressive residual stresses and a work-hardened layer $[18,19]$.

One face of each " $\mathrm{S}$ " Almen strip was shot peened for 2 min using glass beads. Coverage was approximately $95 \%$. The diameter of the beads was between $0.4 \mathrm{~mm}$ and $0.6 \mathrm{~mm}$. The air pressure was $80 \mathrm{psi}$, and the nozzle was $100 \mathrm{~mm}$ from the target. After shot peening, the average deflection at the center of the Almen strip was $0.46 \pm 0.02 \mathrm{~mm}$ (Fig. 2) indicating compressive residual stress was imparted within the sample. Deflection was measured by an Almen gage (Model: TSP-3) of $1 \mu \mathrm{m}$ resolution manufactured by Electronics Inc.

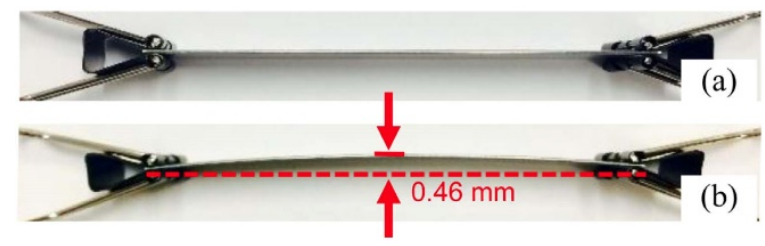

Figure 2. Deflection of (a) as-received ("N") and (b) shot-peened (" $\left.\mathrm{S}^{\prime \prime}\right)$ Almen strips.

\subsection{Stacked Almen strip sequences}

Impact targets consisted of 16 stacked Almen strips of SAE 1070 steel (Type N-1S) from Electronics Inc. Three different hybrid stacking sequences were compared against a stacked control sample (Fig. 3). The red or "S" layers indicated a shot-peened Almen strip. Gray or "N" layers were as-received non-shot-peened Almen strips. The three hybrid stacking sequences were S/NNN (total of 4 shot-peened strips), S/NN (total of 6 shotpeened strips), and $\mathrm{S} / \mathrm{N}$ (total of 8 shot-peened strips). The stiffness of these target surfaces varies based on the number of shot-peened layers and the sequence. 


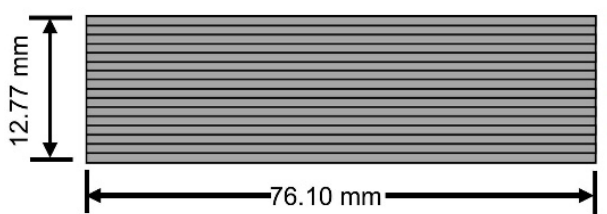

(a)

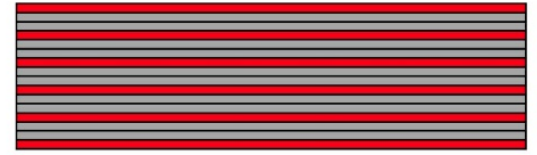

(c)

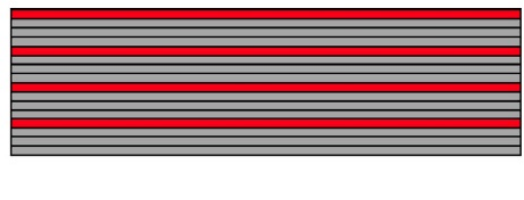

(b)

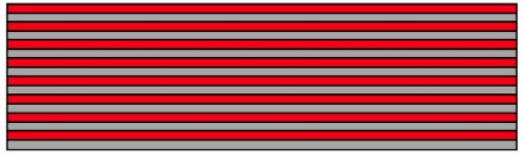

(d)

Figure 3. Configuration of stacked 1070 steel Almen strips with intermittent shot-peened layers: (a) non-shot peened, (b) sequence A (S/NNN), (c) sequence B $(S / N N)$, and (d) sequence $C(S / N)$. " $S$ " indicates shot peened and " $N$ " indicates non-shot peened.

Each individual Almen strip measured $76.10 \mathrm{~mm}$ (length) by $19 \mathrm{~mm}$ (width) by 0.798 $\mathrm{mm}$ (thickness). Two steel c-shaped clamps secured the stacked strips and minimized the gap between layers in order to avoid any spring effects from stacking as shown in Figure 1(b). Once stacked, the thickness of the impact targets were $12.77 \mathrm{~mm}$. The thickness of stacked strips was measured with calipers across the length of the sample with no observable deviations. Therefore, damping due to spring compression of a peened strip was assumed to be negligible.

\subsection{Impact test}

A monorail uniaxial drop tower manufactured by Cadex Inc. was used for low-velocity impact tests to assess energy absorption of hybrid targets. More information regarding uniaxial drop towers can be found in [20]. The drop tower consisted of the following components: (a) a stiff guided free-falling mass (ball arm) of $0.631 \mathrm{~kg}$ that served as the impactor; (b) a uniaxial accelerometer (Model: 44A_00_BCAB) with 5000 Gs capacity mounted within the ball arm at the center of gravity; (c) a flat $125 \mathrm{~mm}$ diameter steel anvil with a thickness of $24 \mathrm{~mm}$; (d) a time gate that measured the drop velocity prior to impact; (e) a Cadex load cell assembly (model: 430_00_LCEL) of $25 \mathrm{kN}$ capacity to measure impact force; and (f) a digital acquisition system. The tower also contained a rebound brake assembly to prevent multiple impacts. The drop carriage in the monorail tower connected to the impactor (ball arm) through a hook. Once the carriage reached the preferred drop height, a switch released the hook and allowed the impactor to freefall toward the target. Since the impactor's shape was a ball, the deformation was nonuniform and aligned to impact the center of the target. The impactor setup and the anvil employed in this study mounted to the uniaxial monorail drop tower as shown in Figure 4. The drop height, drop weight, and drop velocity were $0.5 \mathrm{~m}, 0.631 \mathrm{~kg}$, and $3 \mathrm{~m} / \mathrm{s}$, respectively. Each impact test was repeated six times for each stacking sequence, totaling 24 experiments. The acceleration, load cell, and restitution data were recorded every $30 \mu \mathrm{s}$. 


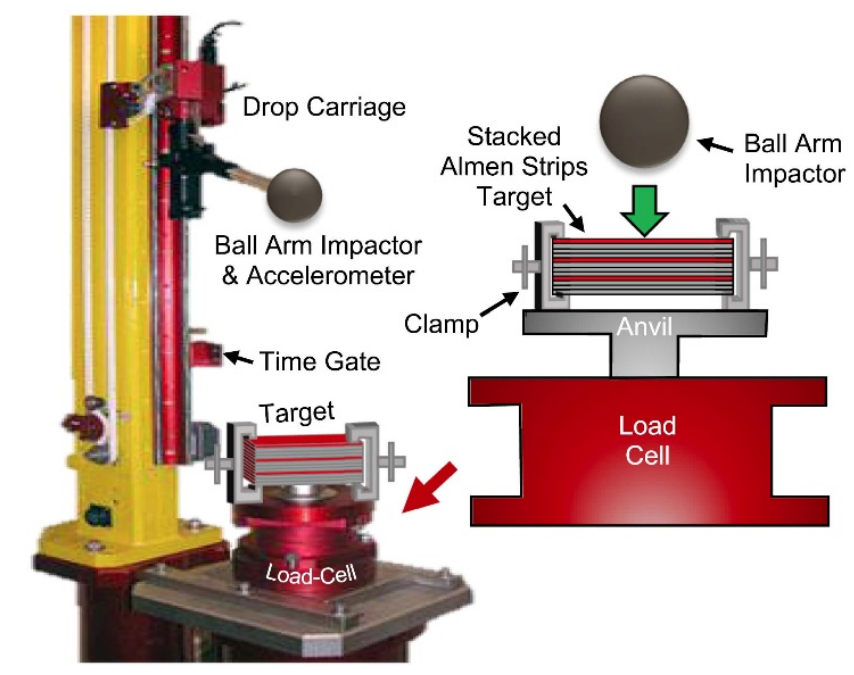

Figure 4. Nonuniform impact tests using a Cadex uniaxial monorail drop tower.

\section{Surface integrity}

Surface integrity of a control (no peening) and shot-peened 1070 steel Almen strip were investigated to further relate the effect of surface roughness, hardness, and residual stress with impact performance and energy absorption. Characterization of surface topography, microhardness, and residual stress is discussed in the following sections.

\subsection{Surface topography}

Keyence laser scanning microscope VK-X200K was used to measure the 3D surface topography of control and shot-peened samples to assess the role friction may have played between peened and control layers on the resulting impact performance. The laser microscope performed noncontact surface profilometry to measure an area of approximately $1.43 \times 1.43 \mathrm{~mm}^{2}$ with a magnification of $10 \times$. The arithmetic mean surface roughness $(\mathrm{Ra})$, root-mean-square roughness $(\mathrm{Rq})$, maximum peak height $(\mathrm{Rp})$, and maximum valley depth (Rv) were analyzed after scanning. Topography was analyzed using VK-Analyzer software.

Figure 5 shows the surface topography profile of the control (no peening) and shotpeened Almen strips. The control strip reveals a typical surface profile after machining with a vertical lay. The shot-peened strip did not exhibit a well-defined pattern. The surface topography metrics are compared in Figure 6. It was observed that Ra did not increase with applying shot peening. This suggests that the effect of roughness and friction between stacked strips on energy absorption is minimal due to the limited change in roughness values resulted from shot peening. However, Rq showed a slight increase after peening. Further, the peak height increased approximately $40 \%$ after shot peening. These measures suggest friction may play a role in energy dissipation of stacked Almen strips. 

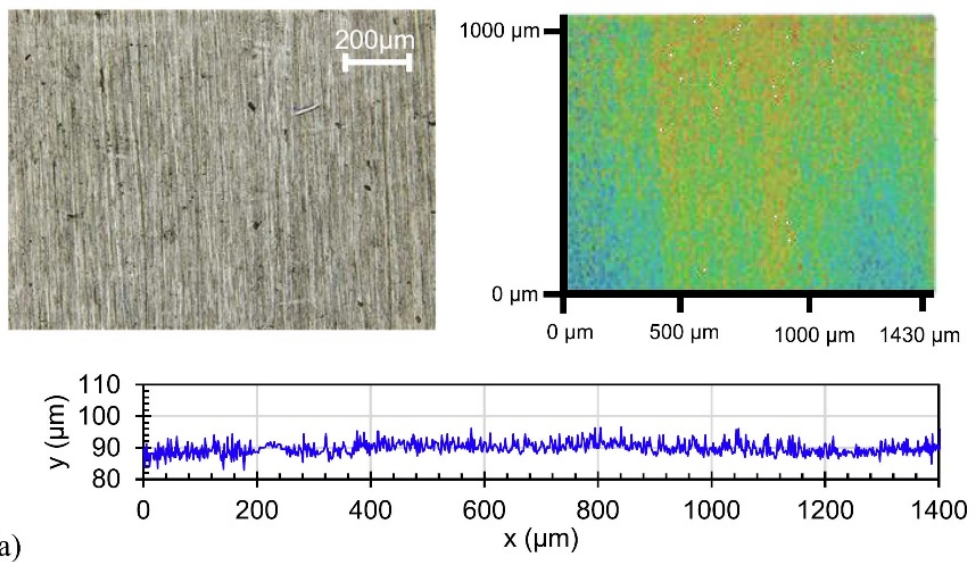

(a)
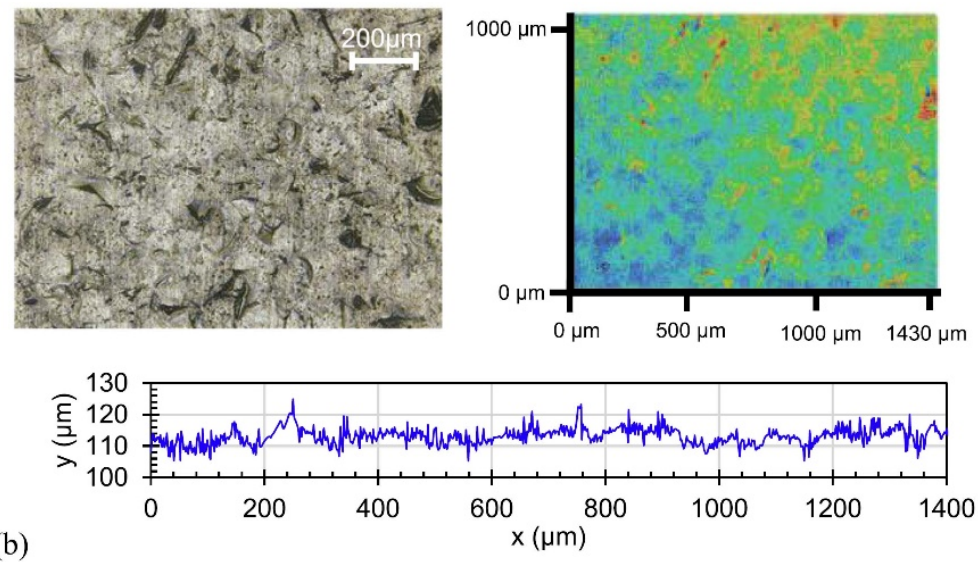

Figure 5. 3D topography profiles of (a) control and (b) shot-peened Almen strips.

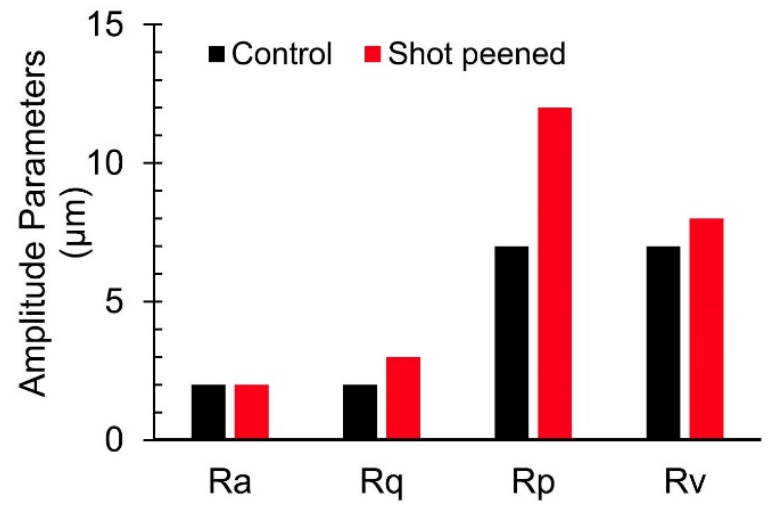

Figure 6. Arithmetic mean (Ra), root-mean-square roughness (Rq), max valley height (Rp), and max valley depth (Rv) of a control and peened 1070 steel Almen strips. 


\subsection{Microhardness}

The Vickers hardness test was used to measure the change in surface hardness of shotpeened compared to a control 1070 steel Almen strips. Microhardness tests were performed using a 200 series Wilson Tukon Microindentor. In this test, A load of 9.8 N (1000 gf) with dwell time of $10 \mathrm{~s}$ was applied along the length of the control and peened strips. The surface hardness for control and peened samples is indicated in Figure 7. It was observed that shot peening the surface of the 1070 steel Almen strip increased the hardness of the surface by about $10 \%$. Thus, this suggests that the accumulation effect of peening a surface with higher intensity or pressure could improve the impact resistance and performance of a component. This 10\% increase is only for one Almen strip (layer); therefore, upon stacking several peened layers (Almen strip) this effect of hardness incrementally influences energy absorption upon low-velocity impact.

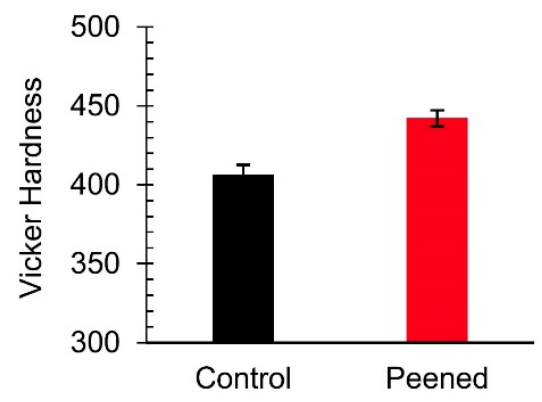

Figure 7. Vicker's microhardness of control (no peening) and shot-peened Almen strips.

\section{Impact results}

The effect of hybrid stacked Almen strips on the impact acceleration and restitution was investigated. Table 1 identifies the average values for hybrid sequences $\mathrm{A}, \mathrm{B}$, and $\mathrm{C}$ as well as the non-shot peened control. Hybrid stacked samples were found to be statistically different from the control target as well as among various stacking sequences. The postimpact rebound height (hr) for the four targets is shown in Figure 8.

Table 1. Nonuniform drop tower impact of shot-peened Almen strips

\begin{tabular}{lccc}
\hline Experiment & $\begin{array}{c}\text { Impact acceleration } \\
\text { Measured }(\mathrm{g})\end{array}$ & $\begin{array}{c}\text { Theoretical } \\
(\mathrm{g})\end{array}$ & $\begin{array}{c}\text { Restitution } \\
(\%)\end{array}$ \\
\hline Non-shot peened & $481 \pm 12$ & 548 & $53.2 \pm 0.9$ \\
Seq. A (S/NNN) & $446 \pm 52$ & 542 & $47.4 \pm 1.4$ \\
Seq. B (S/NN) & $409 \pm 57$ & 507 & $33.4 \pm 8.3$ \\
Seq. C (S/N) & $511 \pm 23$ & 492 & $23.0 \pm 2.6$ \\
\hline
\end{tabular}




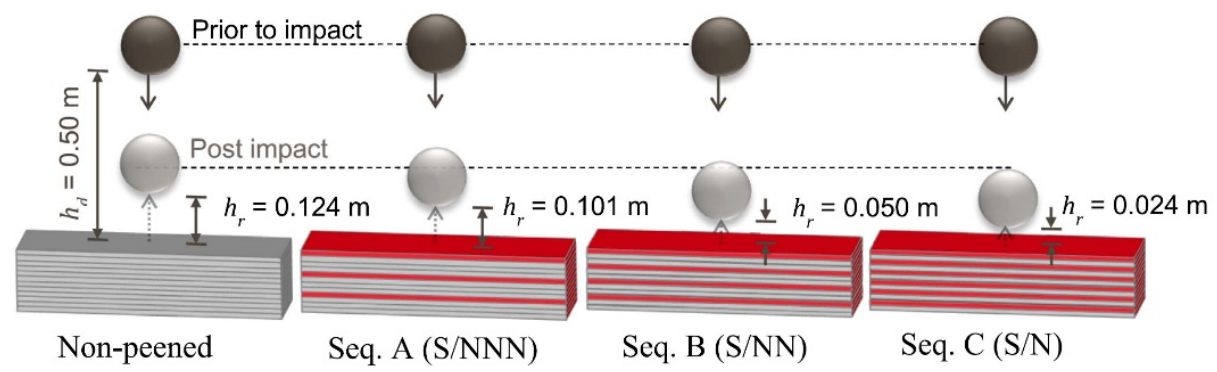

Figure 8. Sequences post-impact behavior.

\subsection{Impact acceleration}

The impact acceleration response depended on the deformation, vibration, and reflection of the impactor-target system, which was primarily influenced by the stiffness and geometry of the target. The results below present the peak impact acceleration and temporal acceleration responses from impacting the ball arm on hybrid stacked Almen strips.

\subsubsection{Peak impact acceleration}

The peak impact acceleration $\left(a_{\text {peak }}\right)$ is a measure of the shock in g-force experienced by the accelerometer mounted within the ball arm's center of gravity and is given by Equation (1). The faster the object changes from a downward velocity to an upward velocity, the greater the peak impact acceleration.

$$
a_{\text {peak }}=\frac{\pi}{T} \sqrt{\frac{g}{2}\left(h_{d}+h_{r}\right)}
$$

From Equation (1), the peak impact acceleration is primarily dependent on two factors: (1) the square root of the drop and rebound heights and (2) the inverse of the pulse width; shorter pulse widths result in higher accelerations. The drop height $\left(h_{d}\right)$ is the pre-impact distance from the bottom of the impactor to the surface of the target. The rebound height $\left(h_{r}\right)$ is the post-impact height as measured from the target's surface to the bottom of the impactor as depicted in Figure 9(a). The pulse width of the impact $(T)$ is the width of the initial peak acceleration verses time curve (Fig. 9(b)), and it is dependent on the shape and material properties of the impactor and the target's surface. 


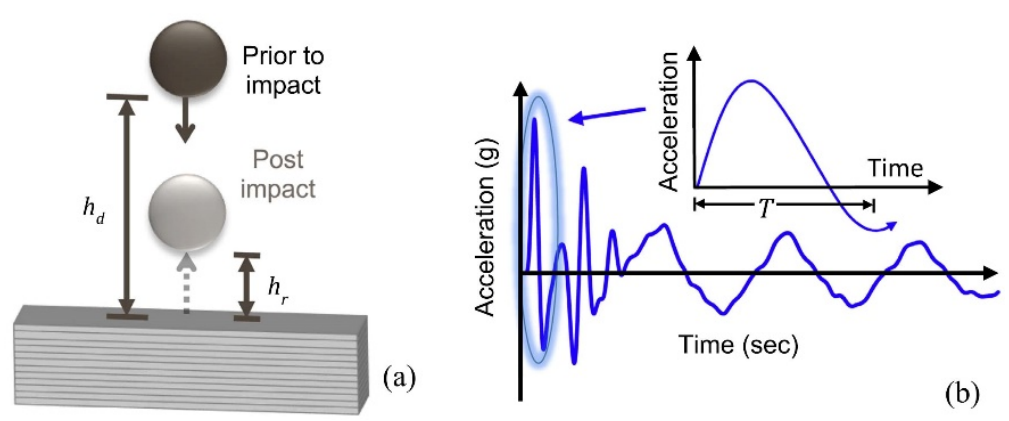

Figure 9. (a) Schematic representation of the pre- and post-impact heights and (b) a representative plot of acceleration versus time from drop impact testing.

Derivation of Peak Acceleration. Acceleration is defined as the rate of change of velocity $(a=\Delta v / \Delta t)$. The term $\Delta v$ is the change from the initial and final velocities, and $\Delta t$ is the impact duration. For impacts, the velocity with respect to time is represented by a sinusoidal function and is given by the following:

$$
v(t)=\left(\frac{v_{1}-v_{2}}{2}\right) \cos \left(\frac{\pi t}{T}\right)+\left(\frac{v_{1}+v_{2}}{2}\right)
$$

The derivative of the velocity yields the acceleration function with respect to time:

$$
a(t)=\frac{d}{d t} v(t)=\frac{d}{d t}\left(\left(\frac{v_{1}-v_{2}}{2}\right) \cos \left(\frac{\pi t}{T}\right)+\left(\frac{v_{1}+v_{2}}{2}\right)\right)=\left(\frac{v_{2}-v_{1}}{2}\right) \frac{\pi}{T} \sin \left(\frac{\pi t}{T}\right)
$$

where the boundary conditions are given by
(a) $a(0)=0$
(b) $a\left(\frac{T}{2}\right)=\frac{\pi}{T}\left(\frac{v_{2}-v_{1}}{2}\right)$
(c) $a(T)=0$

hence,

$$
a_{\text {peak }}=a\left(\frac{T}{2}\right)=\frac{\pi}{T}\left(\frac{v_{2}-v_{1}}{2}\right)
$$

where,

$$
\begin{aligned}
& v_{1}=-\sqrt{2 g h_{d}} \\
& v_{2}=\sqrt{2 g h_{r}}
\end{aligned}
$$

Substituting $v_{1}$ and $v_{2}$ into Equation (4) yields the peak impact acceleration in terms of pulse width, drop height, and rebound height given by Equation (1). 
The experimentally measured mean peak impact acceleration for each stacking sequence is shown in Figure 10. The experiment showed that as the number of shot-peened layers increased, the impact acceleration decreased. This trend held true except for sequence $C(S / N)$, which resulted in a higher linear acceleration of the impactor. This may have been attributed to increased friction from more shot-peened layers. The increased number of layers with higher friction resulted in a higher reaction force upon impact. The increased acceleration on Seq. $C$ may also be explained by a higher stiffness achieved with more peened layers. Hence, the acceleration is highly related to the stiffness of the target surface. The large variation in error bars for Sequences A and B were likely caused by off axis collision and nonuniform peening. This could be improved by automating the peening process and restraining samples to a fixed location with a jig.

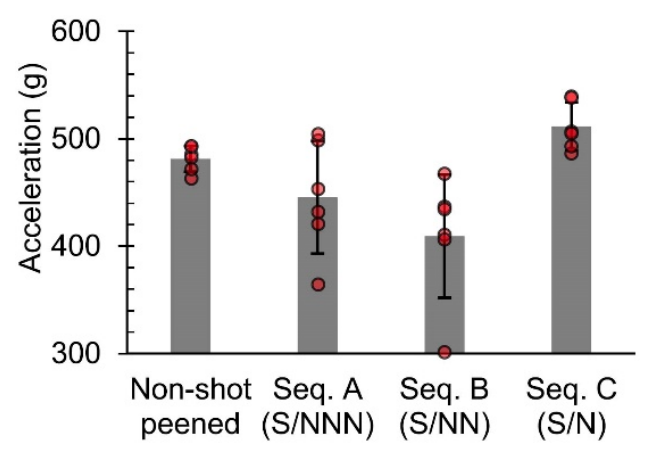

Figure 10. Nonuniform impact acceleration of hybrid stacked 1070 steel Almen strips.

\subsubsection{Temporal acceleration response}

For a better understanding of hybrid stacked shot-peened Almen strip targets, the temporal response for accelerations across sequences were compared over $5 \mathrm{~ms}$ of impact time. Each stacking sequence resulted in different peaks along the $5 \mathrm{~ms}$ temporal profile as shown in Figure 11. Hybrid stacked targets that included shot-peened layers damped the impact acceleration faster than non-shot-peened Almen strip targets. Furthermore, more hybrid layers tended to increase damping within the first $5 \mathrm{~ms}$. 

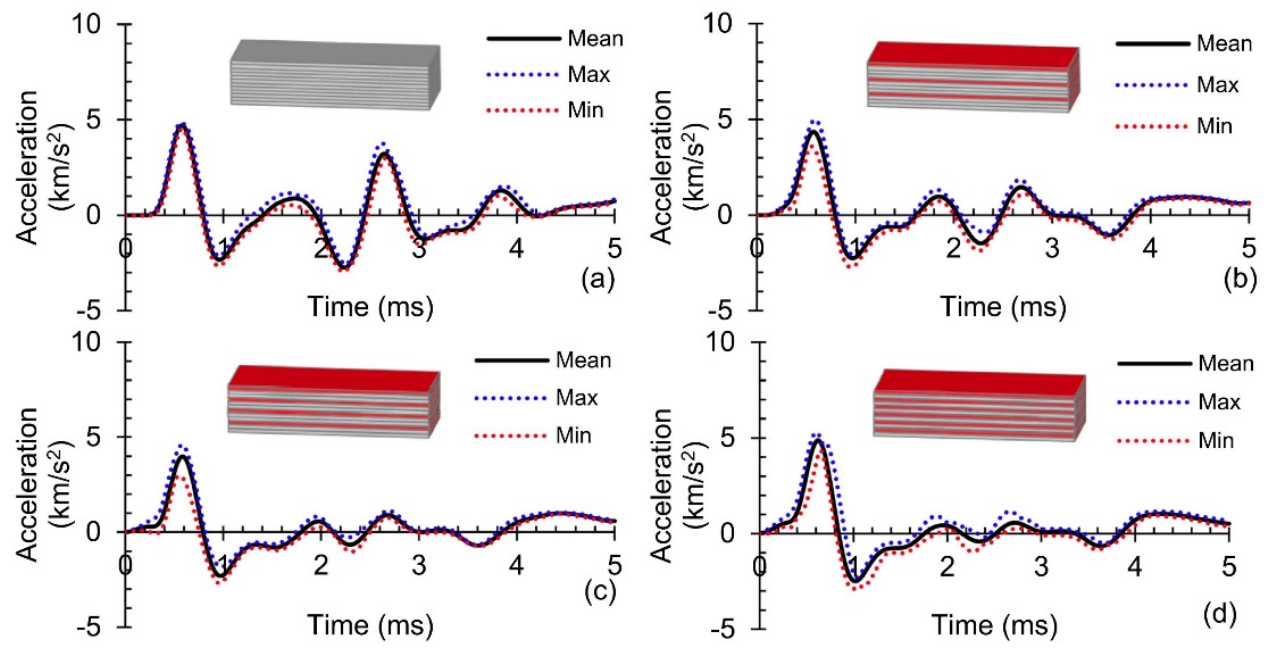

Figure 11. Impact acceleration for (a) non-shot peened, (b) Seq. A (S/NNN), (c) Seq. B $(\mathrm{S} / \mathrm{NN})$, and (d) Seq. C (S/N) stacked Almen strip targets.

The theoretical peak acceleration in Table 1 uses a pulse width corresponding to the width of a complete peak, which was $0.97 \mathrm{~ms}$. The theoretical trend contradicts with the measured trend on Seq. $\mathrm{C}$ by showing a continuously decreasing peak acceleration. This is likely attributed to the fixed pulse width assumed for theoretical values. The percent difference between theoretical and measured ranged between $4 \%$ and $20 \%$.

\subsection{Restitution}

The coefficient of restitution (e) of striking objects is a fractional value representing the ratio of velocities before and after impact. The coefficient of restitution is a measure of how much energy is lost during collision. The restitution ranges between $0 \leq e \leq 1$. In a perfectly plastic collision $(e=0)$, the particles remain together after the collision. In a perfectly elastic collision $(e=1)$, no energy is lost. The coefficient of restitution $(e)$ is defined as

$$
e=\frac{\left(v_{2 \mathrm{~A}}-v_{2 \mathrm{~B}}\right)}{\left(v_{1 \mathrm{~A}}-v_{1 \mathrm{~B}}\right)}
$$

where $v_{2 \mathrm{~A}}$ and $v_{2 \mathrm{~B}}$ are final velocities of objects $\mathrm{A}$ and $\mathrm{B}$, respectively. The terms $v_{1 \mathrm{~A}}$ and $v_{1 \mathrm{~B}}$ are the initial velocities of objects A and B, respectively.

The experimentally determined mean coefficients of restitution for each stacking sequence are shown in Figure 12. The results indicated that the restitution decreased as the number of shot-peened layers included in the stacking sequence increased. Thus, the impactor did not rebound as high on the stacked hybrid samples as it did on the control target. The rebound velocities were significantly lower because the residual stresses caused by shot peening reduced the target's stiffness. Two statistical outliers (out of six experiments) resulted in a relatively large standard deviation for Seq. B and was likely caused by off axis collision or nonuniform peening. 


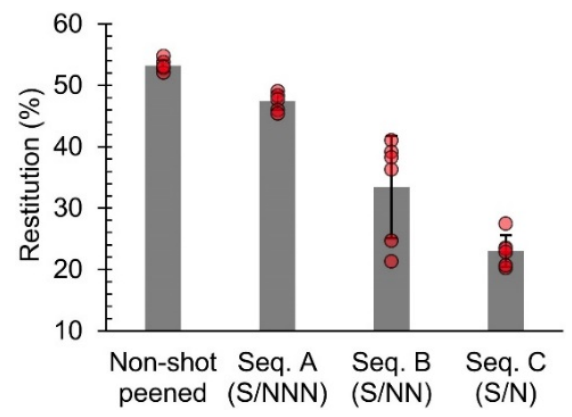

Figure 12. Impact restitution of hybrid stacked 1070 steel Almen strips.

\subsection{Statistical analysis}

Analyses of the variance (ANOVA) were carried out using Minitab software to test the null hypothesis that no difference in the mechanical properties existed among the control and hybrid stacked targets. One-way ANOVA with individual 95\% confidence interval was conducted to test the null hypothesis of both impact acceleration and restitution. After completing ANOVA, Tukey's test with 95\% simultaneous confidence intervals as pairwise comparisons were performed to observe any statistical difference between stacking sequences.

Individual one-way ANOVA for each performance metric was used to either reject or not reject the null hypothesis that the compressive residual stresses provided by shot peening increased the energy absorption capability of hybrid stacked targets. The results of the analyses are provided in Tables 2 and 3. If the $P$-value provided in the following tables is less than $P_{\text {critical }}(0.05$ in this study), then the null hypothesis that the mean values are not different was rejected. That is, the individual treatments provided statistically different outcomes. For $P_{\text {values }}$ greater than $P_{\text {critical, }}$ the null hypothesis fails to be rejected. This means the individual treatments did not produce statistically different results. The pooled standard deviation $(S)$, the coefficient of determination $(R-S q)$, and adjusted $R$-squared ( $R$ $\mathrm{Sq}(\mathrm{adj}))$ are provided in each table.

Table 2. One-way ANOVA of impact acceleration

\begin{tabular}{lccrcc}
\hline Source & DF & SS & MS & F & P \\
\hline Factor & 3 & 35,134 & 11,711 & 7.01 & 0.002 \\
Error & 20 & 33,408 & 1,670 & & \\
Total & 23 & 68,542 & & & \\
\hline
\end{tabular}

Note: $S=40.87, R-\mathrm{Sq}=51.26 \%, R-\mathrm{Sq}(\operatorname{adj})=43.95 \%$

\begin{tabular}{lccccc}
\hline \multicolumn{7}{l}{ Table 3. One-way ANOVA of restitution } & & & \\
\hline Source & DF & SS & MS & F & P \\
\hline Factor & 3 & $3,358.94$ & 1119.6 & 56.86 & 0.000 \\
Error & 20 & 393.8 & 19.7 & & \\
Total & 23 & $3,752.8$ & & & \\
\hline
\end{tabular}

Note: $S=4.438, R-S q=89.51 \%, R-S q(\operatorname{adj})=87.93 \%$ 
Based on statistical analysis, the hybrid stacked Almen strip targets were statistically different from the control for both acceleration and restitution. Furthermore, there were observable differences between different stacking sequences. These differences were analyzed using Tukey's test with an individual confidence level of $98 \%$. Results of the Tukey's test are provided in Table 4 .

Table 4. Tukey's test for stacked Almen strips

\begin{tabular}{|c|c|c|c|c|c|c|c|}
\hline \multicolumn{4}{|c|}{ Impact Acceleration } & \multicolumn{4}{|c|}{ Restitution } \\
\hline Control & Seq. A & Seq. B & Seq. C & Control & Seq. A & Seq. B & Seq. C \\
\hline & & - & & \multicolumn{4}{|c|}{ - } \\
\hline
\end{tabular}

Impact Acceleration: The interpretation of this underscoring is concluding that sequence $A$ and $C$ did not differ from the control. Sequences $A$ and $C$ did not differ from each other, yet sequence B and control differ. Sequences A and B differed from one another as well as sequences B and C. Sequence B had a significantly higher statistical difference in impact acceleration than any of the other sequences.

Restitution: The interpretation of this underscoring is concluding that sequence $\mathrm{A}$ did not differ from the control, yet sequences $B$ and $C$ and control did differ. Sequences $B$ and $\mathrm{C}$ differed compared to sequence $\mathrm{A}$. Furthermore, sequences $\mathrm{B}$ and $\mathrm{C}$ differed and have a statistical difference from one another.

\section{Summary and conclusions}

An experimental investigation on non-uniform, low-velocity impact of stacked 1070 steel Almen strip targets was conducted to determine the influence of integrating shot-peened layers within the stacking sequence on energy absorption. A load cell, accelerometer, and time gate provided impact data that were used to analyze the acceleration of the impactor and the resulting restitution. The effect of stacking sequences was compared to a control nonpeened target. The hybrid stacking sequences under consideration in this study were Seq. A (S/NNN), Seq. B (S/NN), and Seq. C (S/N). The hybrid stacked sequence that offered the lowest restitution absorbed the most energy.

All of the hybrid stacked sequences exhibited favorable performance in terms of energy absorption compared to the control. The results indicated that more shot-peened layers provided better energy absorption. Seq. B that had peening every third layer had the lowest impact acceleration, and Seq. $C$ that had peening every layer had the highest reaction force. This is most likely attributed to a stiffer target. The improvement in impact performance was attributed to shot-peened-induced compressive residual stresses and friction incorporated within predefined layers. In conclusion, the energy absorption of a target can be improved by incorporating enhanced shot-peened layers into the design of a hybridAM target. Future studies will examine the effects of layer sequence and friction on the restitution and acceleration of stacked samples compared to 3D-printed targets that do not exhibit friction between layers. 
CRediT authorship contribution statement $-\mathrm{H}$. Hadidi: Methodology, Investigation, Writingoriginal draft. R. Q. Feng: Methodology, Validation. M. P. Sealy: Conceptualization, Supervision, Project administration, Writing - review and editing.

Declaration of Competing Interest - The authors declare that they have no known competing financial interests or personal relationships that could have appeared to influence the work reported in this paper.

Acknowledgments - The authors would like to acknowledge Jazan University in Saudi Arabia for supporting the lead student investigator through a graduate research fellowship.

\section{References}

[1] Zhang Y, Sun L, Li L, Wang T, Shen L. Experimental and numerical investigations on low-velocity impact response of high strength steel/composite hybrid plate. Int J Impact Eng 2019;123: $1-13$.

[2] Sun G, Chen D, Wang H, Hazell PJ, Li Q. High-velocity impact behaviour of aluminium honeycomb sandwich panels with different structural configurations. Int J Impact Eng 2018;122:11936.

[3] Bianchi G, Aglietti GS, Richardson G. Development of efficient and cost-effective spacecraft structures based on honeycomb panel assemblies. 2010 IEEE aerospace conference. 2010. p. 1-10.

[4] Sealy MP, Madireddy G, Williams RE, Rao P, Toursangsaraki M. Hybrid processes in additive manufacturing. J Manuf Sci Eng 2018;140(6). 060801:1-13.

[5] Randjbaran E, Zahari R, Majid DL, Jalil NAA, Vaghei R, Ahmadi R. The effects of stacking sequence layers of hybrid composite materials in energy absorption under the high velocity ballistic impact conditions: an experimental investigation. J Mater Sci Eng 2013;2(4). 1000130:1-8.

[6] Suresh S. Graded materials for resistance to contact deformation and damage. Science 2001;292 (5526):2447-51.

[7] Li Y, Ramesh KT, Chin ESC. Dynamic characterization of layered and graded structures under impulsive loading. Int J Solids Struct 2001;38(34):6045-61.

[8] Zhang D, Fei Q, Zhang P. Drop-weight impact behavior of honeycomb sandwich panels under a spherical impactor. Compos Struct 2017;168:633-45.

[9] Zhang XC, An LQ, Ding HM. Dynamic crushing behavior and energy absorption of honeycombs with density gradient. J Sandw Struct Mater 2014;16(2):125-47.

[10] Sealy MP, Hadidi H, Kanger CJ, Yan XL, Cui B, McGeough JA. Glocal integrity in 420 stainless steel by asynchronous laser processing. CIRP Ann 2019;68(1):189-92.

[11] Tuan CY. Drop weight testing on sandwich panels with a novel thermoplastic core material. J Sandw Struct Mater 2012;14(3):325-38.

[12] AlMangour B, Yang J. Improving the surface quality and mechanical properties by shot-peening of 17-4 stainless steel fabricated by additive manufacturing. Mater Des 2016;110:914-24.

[13] Sealy MP, Guo YB, Caslaru RC, Sharkins J, Feldman D. Fatigue performance of biodegradable magnesium-calcium alloy processed by laser shock peening for orthopedic implants. Int J Fatigue 2016;82:428-36.

[14] Guo Y, Sealy MP, Guo C. Significant improvement of corrosion resistance of biodegradable metallic implants processed by laser shock peening. CIRP Ann 2012;61(1):583-86. 
[15] Hadidi H, Mailand B, Sundermann T, Johnson E, Madireddy G, Negahban M, et al. Low velocity impact of ABS after shot peening predefined layers during additive manufacturing. Procedia Manuf 2019;34:594-602.

[16] Kanger C, Hadidi H, Akula S, Sandman C, Quint J, Alsunni M, Underwood R, Slafter C, Sonderup J, Spilinek M, Casias J, Rao P, Sealy MP. Effect of process parameters and shot peening on mechanical behavior of ABS parts manufactured by fused filament fabrication (FFF). 2017 Annual International Solid Freeform Fabrication Symposium. 2017. p. 444-58.

[17] Sealy MP, Madireddy G, Li C, Guo YB. Finite element modeling of hybrid additive manufacturing by laser shock peening. Proceedings of the 27th annual international solid freeform fabrication symposium. 2016. p. 306-16.

[18] Kirk D. Estimate compressed layer depth by using Almen peening intensity. Shot Peener 2014; 28(2):30-8.

[19] Hetram, Singh L, Om H. Shot peening effects on material properties: a review. Int J Innov Res Sci Technol 2015;1(12):480-84.

[20] Cadex Inc., 2017. Impact machines, http://www.cadexinc.com, Quebec, Canada. 